\section{Communication course to help dental teams secure success}

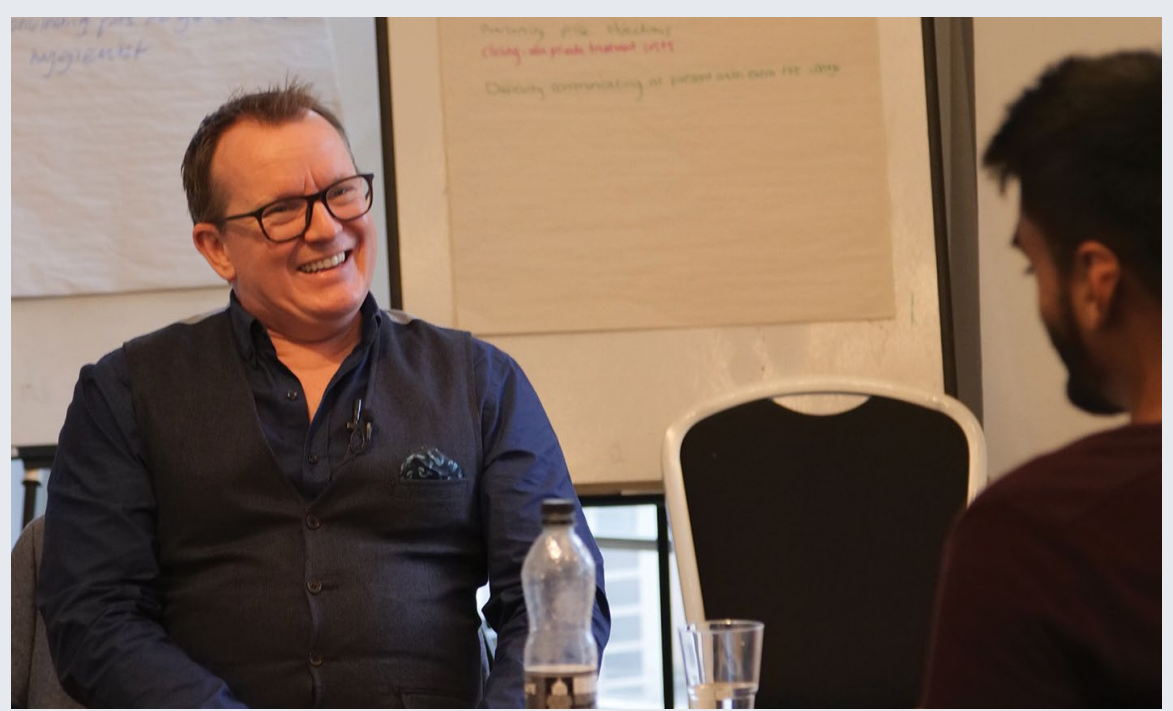

2020 robbed many of us of one of the most fundamental things we crave human contact and connection - with lockdowns and restrictions to our daily lives having a huge impact on mental health and leaving many people feeling isolated, afraid and alone.
As we head into 2021 with yet more uncertainty ahead of us, Dr Barry Oulton (pictured), a highly experienced dentist, lecturer, trainer and businessman, is encouraging dental teams to put building rapport with their patients at the forefront.
His two-day course, Influencing Smiles, is a powerful lesson in communication and sales which teaches principal dentists and their teams how to sell with integrity and communicate with confidence.

It helps dentists boost their confidence and reduce conflict and complaints in their practices at the same time as improving their interactions with patients and boosting treatment uptake by facilitating clearer discussions around options and costs.

Upcoming events for 2021 (depending on COVID restrictions) include:

- 12-13 March - Birmingham

- 14-15 May - London

- 11-12 June - Birmingham

- 24-25 September - London

- 15-16 October - Birmingham.

Courses run from 9 am- 6 pm on Friday and 8 am -5 pm on Saturday.

For further details, visit www. theconfidentdentist.com, call 03332202447 or email sally@theconfidentdentist.com.

\title{
First ever fluoride-containing bioglass toothpaste wins FDA approval
}

British-developed BioMin F is to be available in the USA, known in the US as Dr.Collins BioMin Restore Plus.

Dr.Collins has announced that BioMin Restore Plus Toothpaste has received FDA $510 \mathrm{~K}$ approval for relief of sensitivity. The toothpaste uniquely controls the release of calcium, phosphate and fluoride ions for many hours after brushing to develop acidresistant fluorapatite on the tooth surface and inside exposed dentine tubules.

Available only on prescription from registered dental clinicians, Dr.Collins BioMin Restore Plus is based on BioMin $\mathrm{F}$, the patented bioactive glass complex developed in the laboratories of Queen Mary University of London, UK, by Professor Robert Hill and his team of materials scientists. This award-winning formulation was introduced in Europe in late 2016 and is now available in many regions around the world. Dr.Collins Inc, California, the US licence holder, anticipates the product will be available in the US in spring 2021.

After brushing with BioMin Restore Plus, the active ingredient adheres to the tooth surface, where these microscopic particles slowly dissolve over an 8-12-hour period, constantly releasing calcium, phosphate and fluoride ions. These combine to deposit fluorapatite, similar to natural tooth enamel but more acid-resistant, forming a protective coating on the tooth surfaces. It is also precipitated inside any exposed dentine tubules, plugging them and preventing the fluid flow which causes dentine hypersensitivity.

It is this controlled release mechanism, continuing to deposit mineral on the tooth surface over several hours, which helps Dr.Collins BioMin Restore Plus to be more effective at relieving sensitivity than conventional toothpastes, which are rinsed away in around an hour.

Studies at Queen Mary University have shown that new mineral is created in less than an hour after brushing and the process can continue for up to 12 hours.

Dr.Collins BioMin Restore Plus toothpaste has a fresh mint taste and leaves the mouth feeling clean and healthy. For those who prefer a fluoride-free alternative, Dr.Collins BioMin Restore toothpaste is also available, based on a calcium/phosphate bioglass.

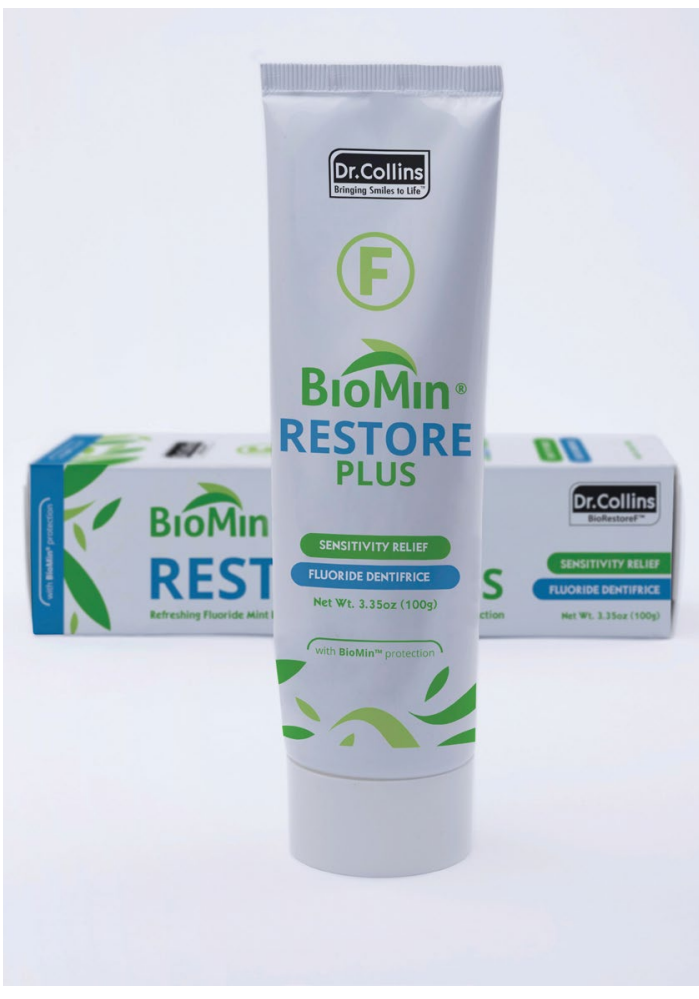

Dr.Collins BioMin Restore and Restore Plus toothpastes are directly available at https://biomintoothpaste.com. 